\title{
Misfit Strain in Superlattices Controlling the Electron-Lattice Interaction via Microstrain in Active Layers
}

\author{
Nicola Poccia, Alessandro Ricci, and Antonio Bianconi \\ Department of Physics, University of Rome "La Sapienza", P. le A. Moro 2, 00185 Roma, Italy \\ Correspondence should be addressed to Antonio Bianconi, antonio.bianconi@roma1.infn.it
}

Received 15 October 2009; Revised 27 November 2009; Accepted 3 December 2009

Academic Editor: Dragan Mihailovic

Copyright () 2010 Nicola Poccia et al. This is an open access article distributed under the Creative Commons Attribution License, which permits unrestricted use, distribution, and reproduction in any medium, provided the original work is properly cited.

\begin{abstract}
High-temperature superconductivity (HTS) emerges in quite different electronic materials: cuprates, diborides, and iron-pnictide superconductors. Looking for unity in the diversity we find in all these materials a common lattice architecture: they are practical realizations of heterostructures at atomic limit made of superlattices of metallic active layers intercalated by spacers as predicted in 1993 by one of us. The multilayer architecture is the key feature for the presence of electronic topological transitions where the Fermi surface of one of the subbands changes dimensionality. The superlattice misfit strain $\eta$ between the active and spacer layers is shown to be a key variable to drive the system to the highest critical temperature $T_{c}$ that occurs at a particular point of the $3 \mathrm{D}$ phase diagram $T_{c}(\delta, \eta)$ where $\delta$ is the charge transfer or doping. The plots of $T_{c}$ as a function of misfit strain at constant charge transfer in cuprates show a first-order quantum critical phase transition where an itinerant striped magnetic phase competes with superconductivity in the proximity of a structural phase transition, that is, associated with an electronic topological transition. The shape resonances in these multigap superconductors is associated with the maximum $T_{c}$.
\end{abstract}

\section{Introduction}

Enormous efforts have been spent since the discovery of high temperature superconductors (HTSs) in 1986 [1] to grab the physics that drives the macroscopic quantum effects from low to high-temperature. After twenty-three years of investigations the community is now looking for a single mechanism of high $T_{c}$ superconductivity emerging in quite different layered systems made of copper oxides $\left(\mathrm{CuO}_{2}\right)$ diborides $\left(\mathrm{B}_{2}\right)$ and iron pnicitdes (FeAs) layers, discovered in 1986, 2001, and 2008, respectively. Understanding the lattice effects that control the critical temperature at constant doping is now considered a key point in the search for unity in the diversity among different HTSs [2-5]. The lattice control of the functional electronic properties has been found in colossal magneto-resistance (CMR) manganites and in the field of ultracold Fermi gases where it has been shown that the Bose or BCS condensation can be controlled by making optical lattices. A first common feature in the field of HTS is understanding the material dependent properties that are now considered a key physical term for understanding HTS. A second universal feature is the multicomponent scenario in the verge of phase separation. In fact in cuprates the polarons $[1,6,7]$ and free carriers coexist where the polarons (in the intermediate regime between small and large polarons) span about $8 \mathrm{Cu}$ sites [8] forming a Wigner crystal at $1 / 8$ doping $[9,10]$ and polaronic $1 \mathrm{D}$ charge density waves [11] that coexist with a free correlated Fermi liquid. The coexistence of polarons [12-16] and free carriers in cuprates is now well established. In fact many experiments show the coexistence of the pseudogap and superconductivity. In fact the pseudo gap is related with polaron ordering both in cuprates and in manganites where they are more close to the small polaron limit [17].

A lot of time has been lost looking for HTS in the proximity to a Mott insulating state, but this feature of cuprate superconductors is not shared with both borides and pnictides. On the contrary a common feature is the proximity to a first-order quantum phase transition where two metals [18-20] with comparable energy compete [21]. For example, in the case of cuprates at optimum doping the attention is addressed now toward the competition of a striped magnetic phase (a polaron Wigner crystal at $1 / 8$ doping) with the superconducting phase as it is observed in oxygen doped $\mathrm{La}_{2} \mathrm{CuO}_{4}[22]$. 
It is possible that the common first-order quantum phase transition, triggering the HTS phase in cuprates, diborides, and pnictides, occurs where the chemical potential of a multiband system is tuned near a electronic topological transition (ETT) from a 2D (or 1D) metal to a 3D (or 2D) metal in only one of its subbands [23-27]. In fact in these conditions the exchange-like pairing between the different electronic components (polarons, in a narrow band near the electronic critical point, and free carriers) shows a shape resonance or Feshbach resonance $[25,26]$ that drives up the $T_{c}$.

In this scenario by tuning the chemical potential near a ETT the electronic, magnetic and elastic interactions drive the system near a lattice instability with a large electronphonon interaction for one electronic portion of the system. In presence of disorder these complex materials systems are expected to show phase separation [28-31] with intrinsic, functional, and connected spatial multiple scales, associated with multiple temporal scales. The source of multiscale phenomena could be the local bonding constraints leading to a framework of coexisting short- and long-range fields and competing orders. In this scenario different experimental techniques probing different spatial and temporal scale provide different landscapes. The orchestrated interplay of several order parameters in these complex materials is believed to be in action with quantum critical fluctuations favouring the entanglement giving a quantum coherence that resists to the decoherence attacks of the high temperature. Several quantum criticalities have been proposed in pnictides [27] and cuprates [28] in a multivariable 3D space where the charge density, a variable determined by lattice effects, and disorder are the main variables driving the system to maximum $T_{c}$.

Billinge and Duxbury [32] have considered the "structural compliance" $\delta_{s}$ the ability of the structure to accommodate two different types of carriers with a different local bond shortening associated with the doped electronic hole. The structural compliance has been defined as $\delta_{s}=$ $\left(r_{b}-r_{f}\right) / r_{b}$ where $r_{b}$ and $r_{f}$ are the lengths of the $\mathrm{Cu}-$ $\mathrm{O}$ bond in the "buckled" and "flat" configurations. The effect of structural compliance on charge ordering in the copper oxygen planes can result in stripe nanostructures. The nonuniform nanoworld of sign varying textures in strain, charge, and magnetization has been investigated also in ferroelastic FE and colossal magnetoresistance CMR materials besides HTS $[30,31]$. The central insight is that under doping a nonlinear lattice perturbation can produce intrinsic inhomogeneities that induce multiscale effects for local lattice integrity constraints. The intercell large strain texturing must be supported by intracell deformations, reflected in bond/angle distribution. It has been proposed that in high $T_{c}$ cuprates the local bonding constraints and the long-range consequences of strongly anisotropic elasticity lead to coexisting short- and long-range forces which results in specific networks of multiple, connected scales. The elasticity self-consistently orders the polarons (producing strong local lattice distortions) into patterns of filaments and clumps. The strength of the local distortion versus the bulk modulus elasticity determines the scales of structural patterns from nano to micron and could explain many experimental results in HTS, CMR and FE materials [31]. Several authors agree on the importance to introduce competitive interaction in order to describe the charge, spin, orbital, and energy configurations in different points of the phase diagram in complex functional materials such as HTS [32]. The JT pairing in the lattice gas model proposed by Miranda et al. [33] shows that the effect of Coulomb interactions between layers can stabilize the size of the charge clusters and make the bipolarons mobile. The calculated DOSs show the existence of up to four gaps, whose origin is due to the energy to break each of the four bonds created during the cluster formations. This multigap model suggests a connection between the scenario of several energy gaps and the local inhomogeneities observed in all HTSs systems. There is agreement that the inhomogeneities arise because all HTS are near a first-order transition tuned by the doping and superlattice misfit strain. It has been proved that without the long-range Coulomb repulsion, the system is unstable with respect to the first order phase transition by direct Monte Carlo simulations [34]. We have to remark that all these scenarios agree that HTS is a particular case of multigap superconductivity [35] both for diborides and for iron pnictides [26, 27].

\section{The Superlattice Misfit Strain in HTS}

There is the consensus that the cuprates showing hightemperature superconductivity, are not three-dimensional (3D) cubic perovskites, like $\mathrm{ABO}_{3}$, but all HTS cuprates belong to the subclass of layered defective perovskites made of a stack of infinite layers of bcc $\mathrm{CuO}_{2}$ layers intercalated by different layers playing the role of spacers [36-39]. This is now a well-established common feature of all known HTSs (cuprates, diborides, and iron pnictides): their lattice architecture is made of stacks of active superconducting planes intercalated by spacers or block layers as shown in Figure 1. For example, the hole-doped oxygen-doped layered perovskite family $\mathrm{La}_{2} \mathrm{CuO}_{4+y}$ is made of $\left[\mathrm{CuO}_{2}\right]_{\infty}^{-2+\delta}$ active bcc layers intercalated by rocksalt $\left[\mathrm{La}_{2} \mathrm{O}_{2+y}\right]_{\infty}^{+2-\delta}$ spacer fcc layers.

The studies of the variation of the superconducting critical temperature $T_{c}$ among cuprates by substitution of cations in the spacers, that have the same valence but different radii, show that their primary effect is a lattice effect which results in dramatic change on $T_{c}$. Therefore there is growing interest on the out-of-plane structural influence (i.e., going on in the spacer layers) that controls the basic intrinsic feature of the electronic structure of $\mathrm{CuO}_{2}$ plane. In fact the maximum $T_{c}$ has been shown to vary widely (by up to a factor of 10) between crystals sharing the same hole density in $\mathrm{CuO}_{2}$ plane but different structures or ordering of dopants in the spacer layers. Therefore the identification of such an out-of-plane influence may be pivotal to finding a roadmap to higher- $T_{c}$ superconductors or manipulation of the superconducting state for novel electronic devices. The chemical pressure is a second variable, beyond doping, that has to be considered to drive the system toward the ETT of 


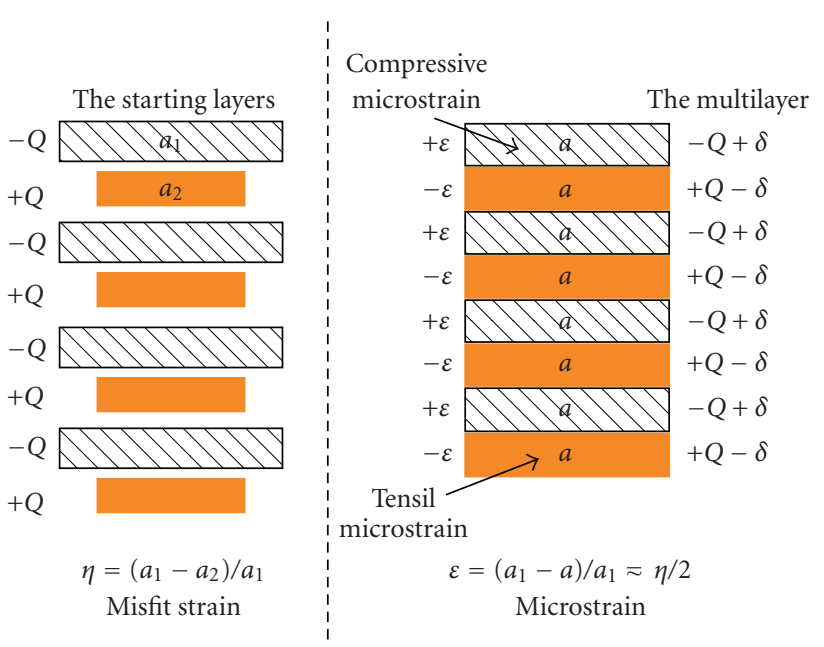

FIgURe 1: Pictorial view of the superlattice with a first layer with a first material characterized by lattice parameter $a_{1}$ and a second layer with a second material characterized by a lattice parameter $a_{2}$. The superlattice misfit strain is $\eta=\left(a_{1}-a_{2}\right) / a$ where $a$ is the mean averaged lattice parameter $a=\left(a_{1}+a_{2}\right) / 2$. The compensated superlattice, showed on the right, has an overall microstrain zero, with $a$ first layer under compressive microstrain $\varepsilon\left(a-a_{1}\right) / a$ and a second intercalated layer under a tensile microstrain $\left(a_{2}-a\right) / a$. It is therefore easy to show, because of the balance in the compensated superlattice of the microstrain, that the misfit strain is related to microstrain by the following relationship $\eta=2 \varepsilon$.

one of its components that will trigger the shape resonance for the high $T_{c}$ in HTS $[25,26]$.

In all $3 \mathrm{D}$ perovskites $\mathrm{ABO}_{3}$ and in manganites, it is well established that the phase diagram of the electronic phases depends on the two variables, charge density and chemical pressure. The chemical pressure is described by the tolerance factor [40]. In perovskites the tolerance factor $t$ has been used to explain the variation of the ratio c/a in the $\mathrm{K}_{2} \mathrm{NiF}_{4}$ structure at very large dopings; in fact for a $t>1$ the atomic displacements parallel to the $c$ axis induce an increase of the $c$ lattice parameter to relieve the compressive stress [41]. For La124 cuprate perovskites with $\mathrm{K}_{2} \mathrm{NiF}_{4}$ structure it has been proposed that the tolerance factor drives the system in a regime of quantum critical magnetic fluctuations [42].

Since HTS superconductors are superlattices of metallic layers, the appropriate physical variable describing elastic effects is not the tolerance factor for $3 \mathrm{D} \mathrm{ABO}_{3}$ systems but the superlattice misfit strain between the different layers [ 43 , 44]. Therefore for the layered superconducting perovskites made of a superlattice of $\mathrm{CuO}_{2}$ layers separated by multiple complex spacer layers the relevant physical quantity is the misfit strain between the $\mathrm{CuO}_{2}$ active and the spacer layers. For layered high- $T_{c}$ superconductors, the superlattice misfit strain should be considered as important as the doping as it was first pointed out first for cuprates [45-48], after for the diborides [49], and finally for pnictides [50].

In cuprates it has not been trivial to introduce the measure of the superlattice misfit strain for because the spacer layers are made of complex different materials with multiple cations having largely different coordination numbers. The measure of the superlattice misfit strain [45] and its introduction in the cuprate phase diagram besides doping and temperature has allowed to quantify the lattice effects in HTS. Increasing the value of the misfit strain above a critical value the systems are driven to a structural phase transition. High $T_{c}$ occurs at low misfit strain but large disorder, dislocations, lattice stripes, and incommensurate lattice modulations appear approaching the structural phase transitions. As we show in Figure 1 the superlattice misfit strain is defined as $\eta=\left(a_{1}-a_{2}\right) / a$, where $a_{1}$ and $a_{2}$ are the unit cell parameters of the ideal first and second layers, respectively, when they are well separated, and $a=$ $\left(a_{1}+a_{2}\right) / 2$. In the compensated multilayer the first layers of the superlattice exhibit a compressive microstrain $\varepsilon_{1 c}=$ $\left(a_{1}-a\right) / a$ and the second layers a tensile microstrain $\varepsilon_{2 t}=$ $\left(a-a_{2}\right) / a$. The average strain is zero in the compensated superlattice, approximating the elastic constants in the active and the intercalated layers as equal, $\varepsilon=\varepsilon_{1 c}=\varepsilon_{2 t}$, and the lattice parameter of the superlattice is close to $\left(a_{1}+a_{2}\right) / 2$. The microstrain can be obtained by measuring the lattice parameter of the superlattice, a, by knowing the unrelaxed ideal lattice parameter of only one of the two layers. The superlattice misfit strain will be given by $\eta=\varepsilon_{1 c}+\varepsilon_{2 t}=2 \varepsilon$ as it was proposed in [45].

Therefore the problem has been solved for cuprates by obtaining the misfit strain from the measure of the compressive microstrain $\varepsilon=\left(R_{0}-r\right) / r$ in the $\mathrm{CuO}_{2}$ plane (that has the same absolute value as the tensile microstrain in the intercalated layers). The determination of the value of the equilibrium $\mathrm{Cu}-\mathrm{O}$ distance for a $\mathrm{Cu}^{2+}$ ion with square planar coordination has been solved using the value $R_{0}=197 \mathrm{pm}$ of the $\mathrm{Cu}^{2+}$ ion in water solution measured by EXAFS. The superlattice misfit strain measuring the elastic field acting on the active and intercalated layers of a superlattice can be therefore obtained by measuring the microstrain in the active layer [45-50].

The microstrain is changed in cuprates and in diborides by chemical substitution of ions with different ionic radii in the spacer layers. The internal chemical pressure acts as a complex anisotropic stress tensor that produces a compressive microstrain of the bcc $\mathrm{CuO}_{2}$ layer in cuprates and a tensile microstrain of the graphene-like B layer in magnesium diborides and of the Fe layers in pnictides.

A strong support for the misfit strain scenario is the behaviour of the spin-gap energy for several different cuprates at constant doping such as $\mathrm{La}_{2-x} \mathrm{Sr}_{\mathrm{x}} \mathrm{CuO}_{4}(x=$ 0.16) (LSCO) $\mathrm{YBa}_{2} \mathrm{Cu}_{3} \mathrm{O}_{6.85}$ (YBCO), $\mathrm{Bi}_{2} \mathrm{Sr}_{2} \mathrm{CaCu}_{2} \mathrm{O}_{8+y}$ (BSCO), and for $\mathrm{La}_{2-x} \mathrm{Sr}_{x} \mathrm{CuO}_{4}$ (LBCO) as shown in Figure 2 [47]. The experimental results show a correlation between magnetic excitations and misfit strain at constant doping $1 / 8$. The cuprates at constant $1 / 8$ doping show a bicritical point at a critical misfit strain $\eta_{c}$ between the superconducting and a static magnetic order. Increasing further the misfit strain the system goes to the structural phase transition from the $T$ to $T^{\prime}$ phase. This trend makes clear that the dynamical magnetic excitations show the typical behaviour in the proximity of a critical point of a quantum phase transition. The high $T_{c}$ superconductivity occurs in the region of a quantum 


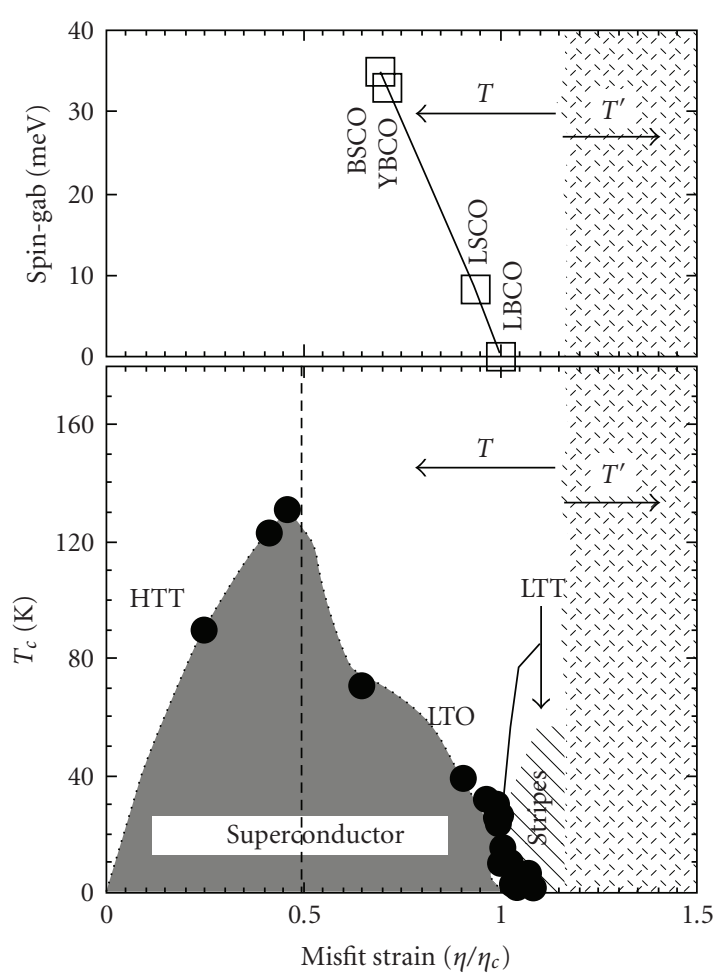

FIGURE 2: Lower panel: spin-gap energy as a function of misfit strain $\eta / \eta_{c}$, normalized to the critical values of the $1 / 8$ phase, in different superconducting cuprates at optimum doping. Upper panel: the superconducting critical temperature as a function of the misfit strain at constant doping $(1 / 8)$ and the Wigner polaron crystal in Nd doped La214 cuprate perovskites. The structural transition from $T \rightarrow T^{\prime}$ at high misfit strain is indicated.

paramagnetism near the onset of quantum fluctuation as shown in Figure 3.

The proposed $3 \mathrm{D}$ phase diagram $T_{c}(\eta, \delta)$ of cuprates [45-48] in Figure 4 shows different regions of phase separation. The phase separation in the range of optimum doping has been reproduced in a recent work by a theoretical two band model $[51,52]$. The phase with "more itinerant" electrons appears at small misfit strain, and the "more localized" and more ordered phase arises at high misfit strain. At doping $1 / 8$ and at a critical misfit strain $\eta_{c}$ a polaron Wigner crystal has been identified where the spin gap goes to zero and a static magnetic order appears $[9,10]$. The stripe fluctuations in space and time and the phase-separated state appears in the proximity of the Wigner crystal and it is possible to move away from it changing the doping or the misfit strain or both.

A strong support to the key role of the misfit-strain variable besides doping and disorder for the phase diagram of HTS comes recently from many experimental results in superconducting pnictides [53]. Recently Cruz et al. [54] have provided support for the key role of misfit strain changing the crystal lattice structure without changing charge carrier density by isoelectronic subtistution in undoped $\mathrm{CeFeAs}_{1-x} \mathrm{P}_{x} \mathrm{O}$. The results show that decreasing the iron-iron distance the system shows a magnetic quantum

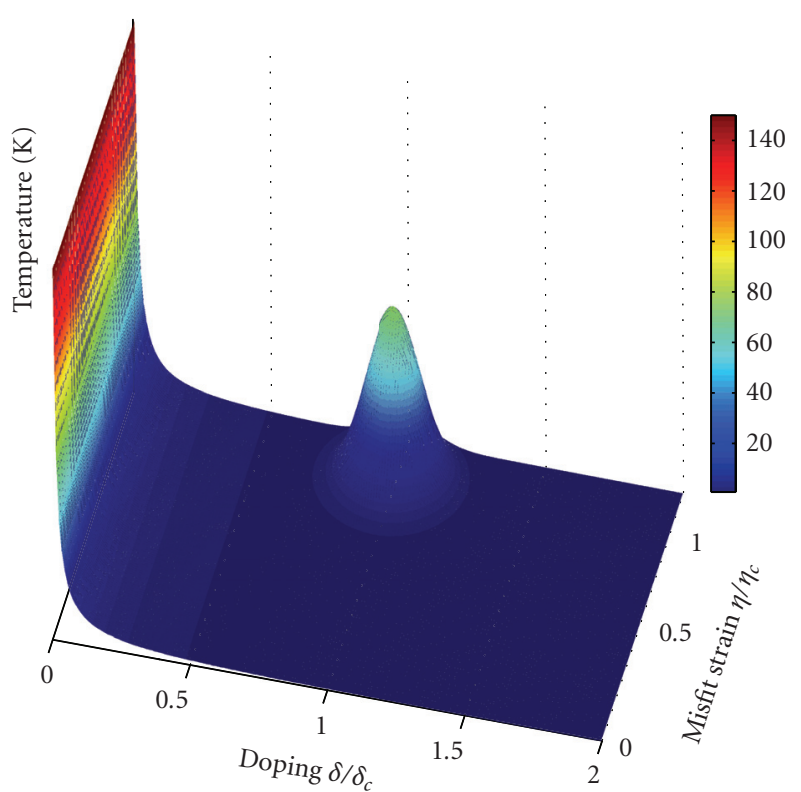

FIgURE 3: The magnetic phase diagram of cuprates: $T_{c}$, doping $\delta$ and misfit strain $\eta$. The The misfit strain $\eta$ (i.e., microstrain or internal chemical pressure) and the doping $\delta$ are normalized to the critical values of the $1 / 8$ phase $\left(\delta_{c}, \eta_{c}\right)$, where the commensurate magnetic stripe phase occurs at $\delta$ doping of $1 / 8$ hole for $\mathrm{Cu}$ site and misfit strain $7 \%$. Therefore the Aeppli-Bianconi critical point is near the point $(1,1)$ in the $2 \mathrm{D}$ space.

critical point where the striped antiferromagnetic order and orthorombic distorsions are suppressed. The work offers additional support for the role of the second axis besides electronic doping in the description of HTS phase diagram. The authors suggest that the pnictogen height in iron arsenide is the important controlling parameter for the electronic and magnetic properties; however it is worth to notice that increasing compressive (tensile) microstrain the As ions are pushed up (down) along the $c$-axis, and therefore the measure of the displacement of the As ion is an indirect measure of the misfit-strain in pnictides.

The misfit-strain can be used to cross a quantum critical point going near the striped phase without the influence of charge carrier doping. It is worth to note that is the same quantum critical state pointed out in cuprates at doping $1 / 8$ and misfit strain 7\% that is shown in Figure 3 [55-57]. Here a first-order quantum critical point occurs where an itinerant striped magnetic phase and superconductivity, in the proximity of a structural phase transition, compete.

In pnictides we have shown that electron-doped FeAs layers have a tensile microstrain due to the misfit strain between the active layers and the spacers. We have identified the critical range of doping and microstrain where the critical temperature gets amplified to its maximum value in Figure 5. The equilibrium $\mathrm{Fe}-\mathrm{Fe}$ distance in the FeAs layers has been found by investigating a set of materials where the intercalated ions in spacer layer have the same charge and plotting the microstrain as a function of ionic radius in the spacer layers. The $\mathrm{Fe}-\mathrm{Fe}$ distance decreases with the ionic 


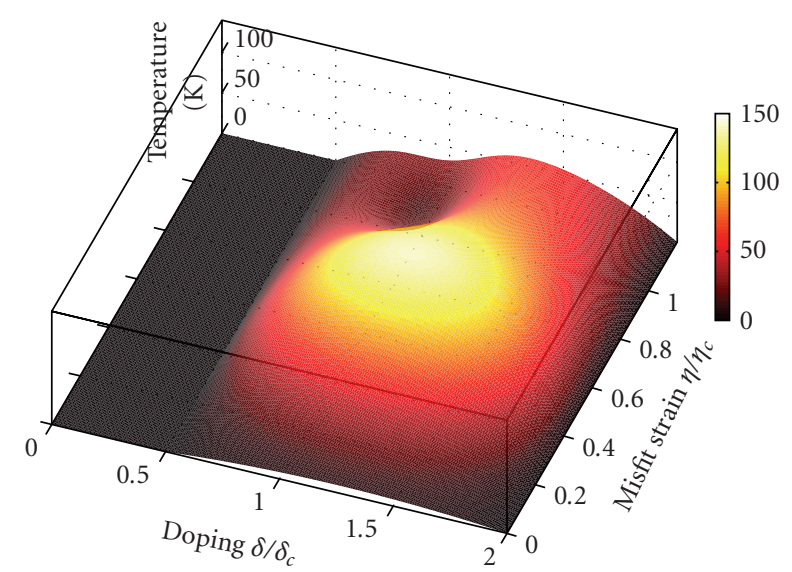

Figure 4: 3D universal phase diagram of the superconducting critical temperature of cuprates. The values of color plot of the superconducting transition temperature $T_{c}$ go from 0 (black) to $135 \mathrm{~K}$ (white). The misfit strain $\eta$ (i.e., microstrain or internal chemical pressure) and the doping $\delta$ are normalized to the critical values of the $1 / 8$ phase $\left(\delta_{c}, \eta_{c}\right)$; therefore the critical point is at $(1,1)$ where the superconducting critical temperature goes to zero (black). The maximum of the critical temperature occurs at (1.4, 0.5 ), that is, at doping 0.16 and misfit strain 0.35 .

radius in the spacers but below a critical radius of the ions in the spacers they do not introduce any variation of the $\mathrm{Fe}-$ Fe distance in the FeAs layer. We have therefore taken the value of the $\mathrm{Fe}-\mathrm{Fe}$ distance in this regime as the unstrained distances for the Fe ions $R_{0}^{-1}=276.35 \mathrm{pm}$ in the $[\mathrm{FeAs}]_{\infty}^{-1}$ layer. The microstrain of the $[\mathrm{FeAs}]_{\infty}^{-1}$ layers can therefore be easily measured. In fact these layers are made of edge sharing FeAs 4 tetrahedral units; therefore the misfit strain induces mainly a rotation of the bonds pushing the As-Fe-As bond out of the ideal value of the tetrahedral angle $109.28^{\circ}$, where the ideal lattice parameter of the orthorhombic lattice is $a_{o}=\sqrt{ } 2 a_{T}=552.7 \mathrm{pm}$.

The phase diagrams for "122" and "1111" pnictides show that the maximum $T_{c}$ occurs in the shaded area of doping and misfit strain shown in Figure 1.

The difference between the region of high $T_{c}$ in "1111" and "122" systems in the misfit-strain doping space is determined by the fact that the two systems are superlattices of quantum wells with very different electronic potential barriers in the spacer layers for itinerant electrons in the active layers.

\section{Conclusion}

We have discussed the superlattice misfit strain as a key material dependent parameter besides doping and temperature for the phase diagram of HTS materials. We have proposed the superlattice misfit strain as the key parameter controlling the elastic field effects in the system that allow to describe the HTS phase diagram for cuprates, magnesium diborides, and iron arsenides. These systems are driven to the point of maximum $T_{c}$ by all or one of the key variables doping, disorder and misfit strain.

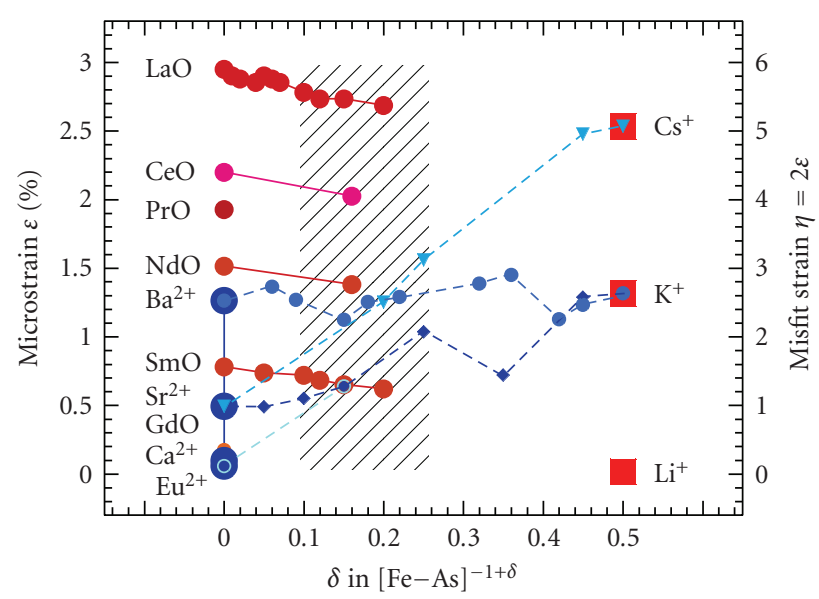

Figure 5: The microstrain $\varepsilon$ (proportional to the Fe-Fe distance) and the misfit strain $\eta=2 \varepsilon$ are plotted as a function of the doping for the " 122 " family and for the " 1111 " family of high-temperature superconducting FeAs compounds. The highlighted area is the region where the maximum $T_{c}$ occurs.

In hole-doped superconductors the compressive strain in the $\mathrm{CuO}_{2}$ plane is related with the tensile $\mathrm{RE}-\mathrm{O}$ microstrain on the rocksalt spacer layer. The different lattice misfit between the building blocks of the different perovskites induces a microstrain on the $\mathrm{CuO}_{2}$ lattice forming short range dynamic lattice stripes with a modulated the $\mathrm{Cu}-\mathrm{O}$ distance in the plane in the range of misfit strain $0.5<$ $\eta_{c}<1$. Electron- doped cuprates and iron arsenides show a tensile microstrain exerted on the $\mathrm{CuO}_{2}$ or FeAs plane and a compressive microstrain on the fluorite spacer layers.

Finally the misfit strain provides a new insight in the complex physics of the HTS systems. A deep understanding of this point will help to material design of new high $T_{c}$ superconductors $[23,37]$ with fascinating and exotic properties for novel technological applications.

\section{References}

[1] J. G. Bednorz and K. A. Müller, "Perovskite-type oxidesthe new approach to high- $T_{c}$ superconductivity," Reviews of Modern Physics, vol. 60, no. 3, pp. 585-600, 1988.

[2] S. A. Kivelson and H. Yao, "Iron-based superconductors: unity or diversity?” Nature Materials, vol. 7, no. 12, pp. 927-928, 2008.

[3] J. Zaanen, "Condensed-matter physics: the pnictide code," Nature, vol. 457, no. 7229, pp. 546-547, 2009.

[4] A. Bianconi, N. Poccia, and A. Ricci, "Unity in the diversity," Journal of Superconductivity and Novel Magnetism, vol. 22, no. 6, pp. 527-528, 2009.

[5] J. Annett, F. Kusmartsev, and A. Bianconi, "Anisotropic and multiband pairing: from borides to multicomponent superconductivity," Superconductor Science and Technology, vol. 22, no. 1, Article ID 010301, 2009.

[6] A. S. Alexandrov, J. Ranninger, and S. Robaszkiewicz, "Bipolaronic superconductivity: thermodynamics, magnetic properties, and possibility of existence in real substances," Physical Review B, vol. 33, no. 7, pp. 4526-4542, 1986. 
[7] A. S. Alexandrov and A. B. Krebs, "Polarons in hightemperature superconductors," Soviet Physics Uspekhi, vol. 35, no. 5, pp. 345-383, 1992.

[8] A. Bianconi, M. Missori, H. Oyanagi, et al., "The measurement of the polaron size in the metallic phase of cuprate superconductors," Europhysics Letters, vol. 31, no. 7, pp. 411-415, 1995.

[9] A. Bianconi and M. Missori, "The instability of a 2D electron gas near the critical density for a Wigner polaron crystal giving the quantum state of cuprate superconductors," Solid State Communications, vol. 91, no. 4, pp. 287-293, 1994.

[10] A. Bianconi, "On the Fermi liquid coupled with a generalized wigner polaronic CDW giving high $T_{c}$ superconductivity," Solid State Communications, vol. 91, no. 1, pp. 1-5, 1994.

[11] A. Bianconi, N. L. Saini, A. Lanzara, et al., "Determination of the local lattice distortions in the $\mathrm{CuO}_{2}$ plane of $\mathrm{La}_{1.85} \mathrm{Sr}_{0.15} \mathrm{CuO}_{4}$," Physical Review Letters, vol. 76, no. 18, pp. 3412-3415, 1996.

[12] A. S. Alexandrov and N. F. Mott, Polarons and Bipolarons, World Scientific, Singapore, 1996.

[13] A. Lanzara, G.-M. Zhao, N. L. Saini, et al., "Oxygenisotope shift of the charge-stripe ordering temperature in $\mathrm{La}_{2-x} \mathrm{Sr}_{x} \mathrm{CuO}_{4}$ from X-ray absorption spectroscopy," Journal of Physics: Condensed Matter, vol. 11, no. 48, pp. L541-L546, 1999.

[14] D. Mihailovic, T. Mertelj, and K. A. Müller, " $a-b$ plane optical conductivity in $\mathrm{YBa}_{2} \mathrm{Cu}_{3} \mathrm{O}_{7-\delta}$ above and below $T^{*}$," Physical Review B, vol. 57, no. 10, pp. 6116-6120, 1998.

[15] A. S. Alexandrov, "Polaron dynamics and bipolaron condensation in cuprates," Physical Review B, vol. 61, no. 18, pp. 1231512327, 2000.

[16] A. Bussmann-Holder and H. Keller, "Polaron formation as origin of unconventional isotope effects in cuprate superconductors," European Physical Journal B, vol. 44, no. 4, pp. 487490, 2005.

[17] A. Lanzara, N. L. Saini, M. Brunelli, et al., "Crossover from large to small polarons across the metal-insulator transition in manganites," Physical Review Letters, vol. 81, no. 4, pp. 878$881,1998$.

[18] R. B. Laughlin, G. G. Lonzarich, P. Monthoux, and D. Pines, "The quantum criticality conundrum," Advances in Physics, vol. 50, no. 4, pp. 361-365, 2001.

[19] P. Coleman and A. J. Schofield, "Quantum criticality," Nature, vol. 433, no. 7023, pp. 226-229, 2005.

[20] P. Goswami, D. Schwab, and S. Chakravarty, "Rounding by disorder of first-order quantum phase transitions: emergence of quantum critical points," Physical Review Letters, vol. 100, no. 1, Article ID 015703, 4 pages, 2008.

[21] C. Castellani, C. Di Castro, and M. Grilli, "Stripe formation: a quantum critical point for cuprate superconductors," Journal of Physics and Chemistry of Solids, vol. 59, no. 10-12, pp. 16941698, 1998.

[22] S. A. Kivelson, G. Aeppli, and V. J. Emery, "Thermodynamics of the interplay between magnetism and high-temperature superconductivity," Proceedings of the National Academy of Sciences of the United States of America, vol. 98, no. 21, pp. 11903-11907, 2001.

[23] A. Bianconi, "Process of increasing the critical temperature $T_{c}$ of a bulk superconductor by making metal heterostructures at the atomic limit," United State Patent no. :US6, 265, 019 B1, July 2001.

[24] A. Bianconi, N. L. Saini, T. Rossetti, et al., "Stripe structure in the $\mathrm{CuO}_{2}$ plane of perovskite superconductors," Physical Review B, vol. 54, no. 17, pp. 12018-12021, 1996.
[25] A. Bianconi, A. Valletta, A. Perali, and N. L. Saini, "Superconductivity of a striped phase at the atomic limit," Physica C, vol. 296, no. 3-4, pp. 269-280, 1998.

[26] A. Bianconi, "Feshbach shape resonance in multiband superconductivity in heterostructures," Journal of Superconductivity, vol. 18, no. 5-6, pp. 25-36, 2005.

[27] R. Caivano, M. Fratini, N. Poccia, et al., "Feshbach resonance and mesoscopic phase separation near a quantum critical point in multiband FeAs-based superconductors," Superconductor Science and Technology, vol. 22, no. 1, Article ID 014004, 12 pages, 2009.

[28] A. R. Bishop, "HTC oxides: a collusion of spin, charge and lattice," Journal of Physics: Conference Series, vol. 108, no. 1, Article ID 012027, 8 pages, 2008.

[29] A. R. Bishop, T. Lookman, A. Saxena, and S. R. Shenoy, "Elasticity-driven nanoscale texturing in complex electronic materials," Europhysics Letters, vol. 63, no. 2, pp. 289-295, 2003.

[30] K. H. Ahn, T. Lookman, and A. R. Bishop, "Strain-induced metal-insulator phase coexistence in perovskite manganites," Nature, vol. 428, no. 6981, pp. 401-404, 2004.

[31] T. Lookman, S. R. Shenoy, K. Ø. Rasmussen, A. Saxena, and A. R. Bishop, "Ferroelastic dynamics and strain compatibility," Physical Review B, vol. 67, no. 2, Article ID 0241142, 27 pages, 2003.

[32] S. J. L. Billinge and P. M. Duxbury, "Structural compliance, misfit strain, and stripe nanostructures in cuprate superconductors," Physical Review B, vol. 66, no. 6, Article ID 064529, 4 pages, 2002.

[33] J. Miranda, T. Mertelj, V. V. Kabanov, and D. Mihailovic, "Bipolaron Jahn-Teller pairing and charge transport in cuprates," Journal of Superconductivity and Novel Magnetism, vol. 22, no. 3, pp. 281-285, 2009.

[34] T. Mertelj, V. V. Kabanov, J. M. Mena, and D. Mihailovic, "Selforganization of charged particles on a two-dimensional lattice subject to anisotropic Jahn-Teller-type interaction and threedimensional Coulomb repulsion," Physical Review B, vol. 76, no. 5, Article ID 054523, 9 pages, 2007.

[35] N. Kristoffel, P. Rubin, and T. Örd, "Multiband model of cuprate superconductivity," International Journal of Modern Physics B, vol. 22, no. 30, pp. 5299-5327, 2008.

[36] Y. Tokura and T. Arima, "New classification method for layered copper oxide compounds and its application to design of new high $T_{c}$ superconductors," Japanese Journal of Applied Physics, vol. 29, no. 11, pp. 2388-2402, 1990.

[37] A. Bianconi, "On the possibility of new high $T_{c}$ superconductors by producing metal heterostructures as in the cuprate perovskites," Solid State Communications, vol. 89, no. 11, pp. 933-936, 1994.

[38] C. N. R. Rao and A. K. Ganguli, "Structure-property relationships in superconducting cuprates," Chemical Society Reviews, vol. 24, no. 1, pp. 1-7, 1995.

[39] K. A. Müller, "On the superconductivity in hole doped cuprates," Journal of Physics: Condensed Matter, vol. 19, no. 25, Article ID 251002, 13 pages, 2007.

[40] C. Li, K. C. K. Soh, and P. Wu, "Formability of $\mathrm{ABO}_{3}$ perovskites," Journal of Alloys and Compounds, vol. 372, no. 1-2, pp. 40-48, 2004.

[41] K. K. Singh, P. Ganguly, P. P. Edwards, and J. B. Goodenough, "Effect of percolation in an intergrowth structure," Journal of Physics: Condensed Matter, vol. 3, no. 15, pp. 2479-2497, 1991.

[42] G. Aeppli, T. E. Mason, S. M. Hayden, H. A. Mook, and J. Kulda, "Nearly similar magnetic fluctuations in the normal 
state of a high- $T_{c}$ cuprate superconductor," Science, vol. 278, no. 5342, pp. 1432-1435, 1997.

[43] G. Forgacs, R. Lipowsky, and T. M. Nieuwenhuizen, "The behaviour of the interfaces in ordered and disordered system," in Phase Transitions and Critical Phenomena, C. Domb and J. L. Lebowitz, Eds., vol. 14, pp. 135-367, Academic Press, London, UK, 1991.

[44] P. Bak, "Commensurate phases, incommensurate phases and the devil's staircase," Reports on Progress in Physics, vol. 45, no. 6, pp. 587-629, 1982.

[45] A. Bianconi, G. Bianconi, S. Caprara, D. Di Castro, H. Oyanagi, and N. L. Saini, "The stripe critical point for cuprates," Journal of Physics: Condensed Matter, vol. 12, no. 50, pp. 10655-10666, 2000.

[46] S. Agrestini, N. L. Saini, G. Bianconi, and A. Bianconi, "The strain of $\mathrm{CuO}_{2}$ lattice: the second variable for the phase diagram of cuprate perovskites," Journal of Physics A, vol. 36, no. 35, pp. 9133-9142, 2003.

[47] M. Fratini, N. Poccia, and A. Bianconi, "The Feshbach resonance and nanoscale phase separation in a polaron liquid near the quantum critical point for a polaron Wigner crystal," Journal of Physics: Conference Series, vol. 108, no. 1, Article ID 012036, 13 pages, 2008.

[48] N. Poccia and M. Fratini, "The misfit strain critical point in the 3D phase diagrams of cuprates," Journal of Superconductivity and Novel Magnetism, vol. 22, no. 3, pp. 299-303, 2009.

[49] S. Agrestini, D. Di Castro, M. Sansone, et al., "High $T_{c}$ superconductivity in a critical range of micro-strain and charge density in diborides," Journal of Physics: Condensed Matter, vol. 13, no. 50, pp. 11689-11695, 2001.

[50] A. Ricci, N. Poccia, G. Ciasca, M. Fratini, and A. Bianconi, "The microstrain-doping phase diagram of the iron pnictides: heterostructures at atomic limit," Journal of Superconductivity and Novel Magnetism, vol. 22, no. 6, pp. 589-593, 2009.

[51] K. I. Kugel, A. L. Rakhmanov, A. O. Sboychakov, F. V. Kusmartsev, N. Poccia, and A. Bianconi, "A two-band model for the phase separation induced by the chemical mismatch pressure in different cuprate superconductors," Superconductor Science and Technology, vol. 22, no. 1, Article ID 014007, 7 pages, 2009.

[52] K. I. Kugel, A. L. Rakhmanov, A. O. Sboychakov, N. Poccia, and A. Bianconi, "Model for phase separation controlled by doping and the internal chemical pressure in different cuprate superconductors," Physical Review B, vol. 78, no. 16, Article ID 165124, 7 pages, 2008.

[53] M. Fratini, R. Caivano, A. Puri, et al., "The effect of internal pressure on the tetragonal to monoclinic structural phase transition in ReOFeAs: the case of NdOFeAs," Superconductor Science and Technology, vol. 21, no. 9, Article ID 092002, 4 pages, 2008.

[54] C. de la Cruz, W. Z. Hu, S. Li, et al., "Lattice distortion and magnetic quantum phase transition in $\mathrm{CeFeAs}_{1-x} \mathrm{P}_{x} \mathrm{O}$," Physical Review Letters, vol. 104, Article ID 017204, 4 pages, 2010.

[55] A. Bianconi, N. L. Saini, S. Agrestini, D. Di Castro, and G. Bianconi, "The strain quantum critical point for superstripes in the phase diagram of all cuprate perovskites," International Journal of Modern Physics B, vol. 14, no. 29-31, pp. 3342-3355, 2000, http://dx.doi.org/doi:10.1142/S0217979200003812.

[56] A. Bianconi, S. Agrestini, G. Bianconi, D. Di Castro, and N. L. Saini, "A quantum phase transition driven by the electron lattice interaction gives high tc superconductivity," Journal of Alloys and Compounds, vol. 317-318, no. 1-2, pp. 537-541, 2001, http://dx.doi.org/10.1016/S0925-8388(00)01383-9.
[57] D. Di Castro, M. Colapietro, G. Bianconi, "Metallic stripes in oxygen doped 12cuo4," Int. J. Mod. Phys., vol. 14, no. 29/31, pp. 3438-3443, 2000, http://dx.doi.org/ doi:10.1142/S0217979200003927. 

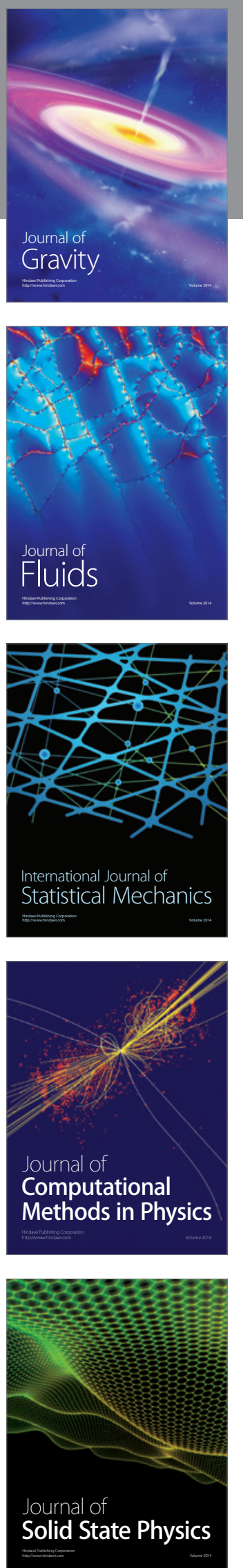

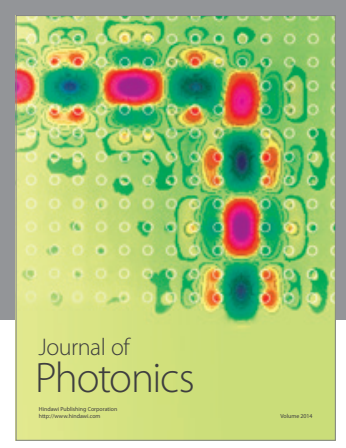

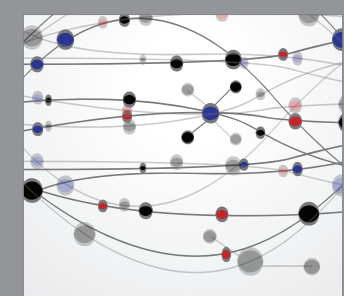

The Scientific World Journal
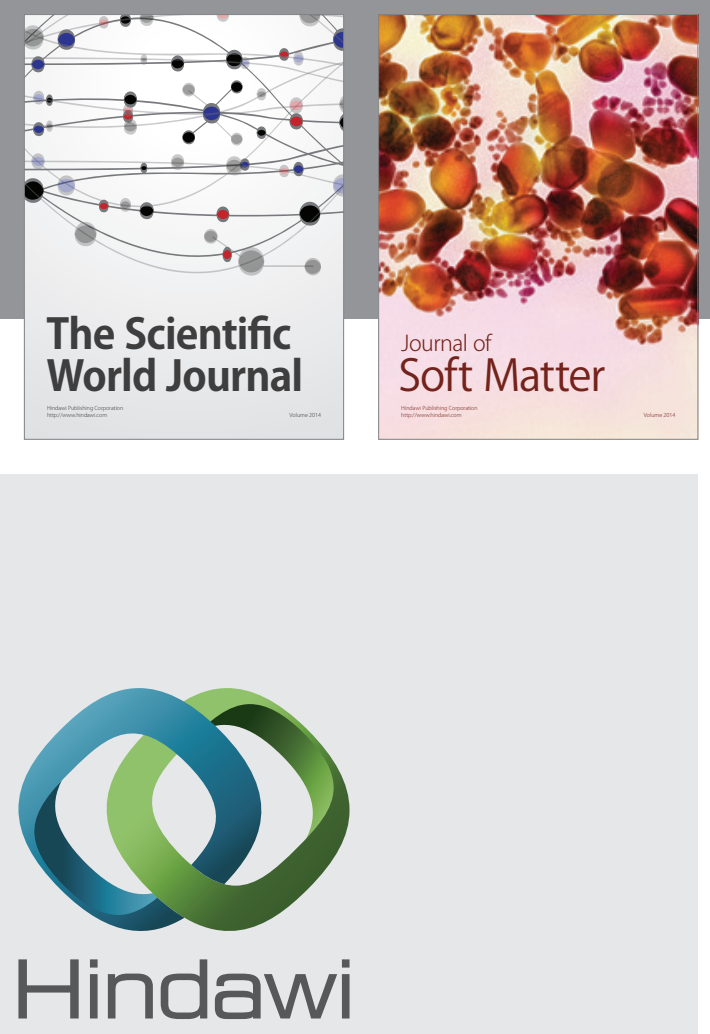

Submit your manuscripts at

http://www.hindawi.com
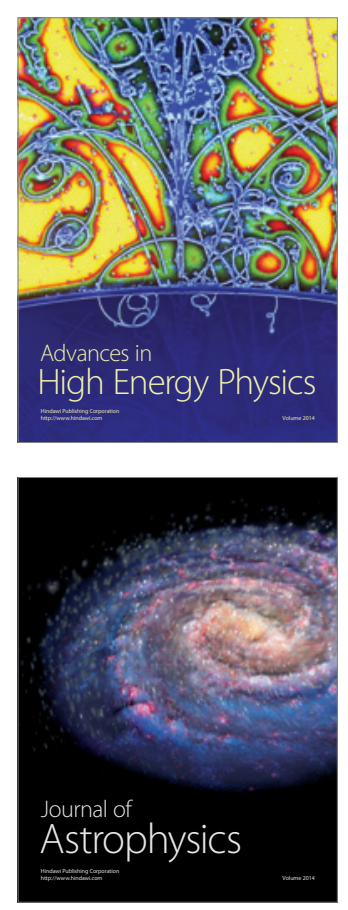
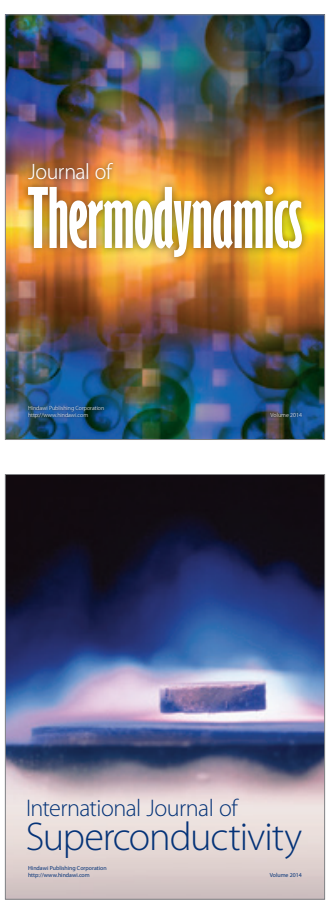
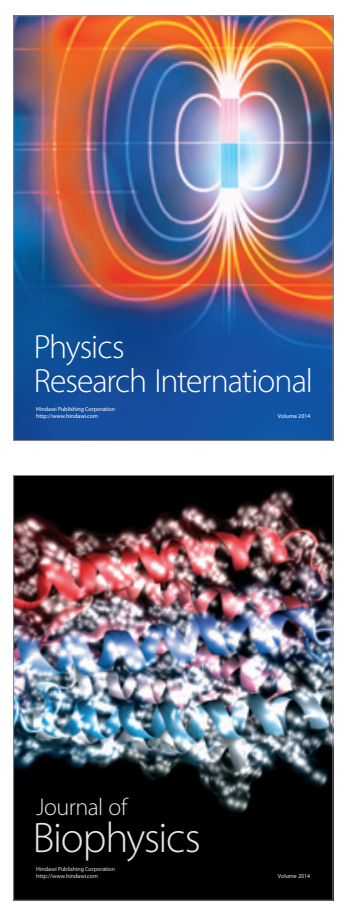
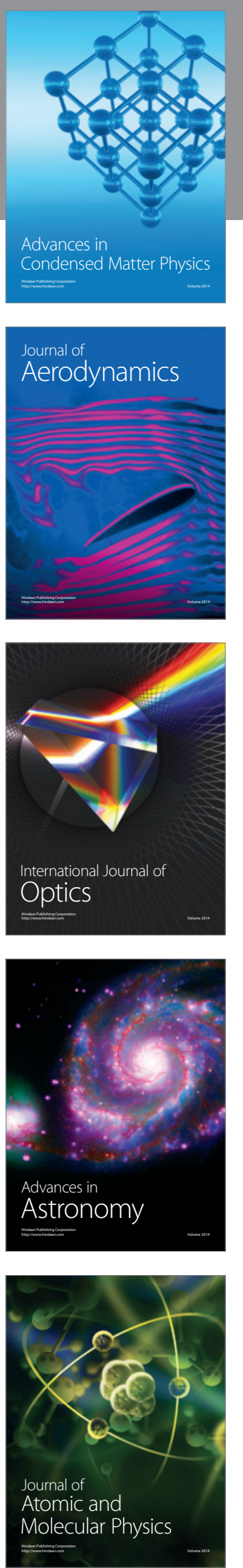